\title{
No experience necessary?
}

\author{
Richard Jeremy Stone
}

When faced with advertisements for astronauts my reaction is to look for their date. This not being the first of a particular month, however, I was intrigued to read further. Medic, scientist, or engineer required, aged 21 to 40 , with proved foreign language ability. It seems many, many months since I replied to this curious challenge. In that time I have reflected more than once (although usually momentarily) if the decision to reply was not a rash one.

Let me provide the background: aged 24 and recently qualified, with a faltering knowledge of a few European languages plus a smattering of research. Not perhaps the strongest qualifications for Britain's first astronaut, but Project Juno (the first Anglo-Soviet space mission) thought otherwise. Thus prepared and with cloudy childhood memories of Armstrong $e t$ al I embarked eagerly on stage one of the selection procedure.

I have always nurtured a sneaking suspicion of IQ tests (IQ being that variable measured by the tests and not much else), but tested we were. My 150 fellow candidates, whittled down from 3500 serious applicants, seemed remarkably able and self possessed while I felt the sweat rise with progressively more complex tests of spatial, mechanical, and logical reasoning. These finished, an afternoon of "executive" medicals followed, and I left London having thoroughly enjoyed an unusual day. Reflecting on average performances throughout, I travelled home proudly clutching

BrMed f 1990;300:27-8 the mission tee shirt, expecting my extraterrestrial ambitions to sink quite rapidly in the West.

Not so; the field narrowed to 35 , and we reconvened three weeks later for briefing and to face a curious press at the Royal Aeronautical Society. One other doctor and I were included on the shortlist. After we had been lectured by lawyers, public relations consultants, sponsorship advisers, and doctors the entire concept seemed no longer surreal. What was eminently tangible was the prospect of highly invasive mental and physical tests. A full day of psychological screening ensued, investigating personality, motivation, and ability to face the press (the chosen man or woman having to expect fascination from the media for many years to come).

Numbers were cut still further to 22, who would undergo more stringent examination. My annual leave request form no doubt made bizarre reading; "five days required for astronaut medicals." The Russians had specified several investigations that to my mind were not only of questionable clinical relevance but moreover were ethically doubtful. We submitted, however, to a barium meal examination and enema, sigmoidoscopy, and prostatic massage, borne with good humour and mild embarrassment. We plodded the quality streets of London from consulting room to consulting room, variously myopic from tropicamide and dizzy from vestibular tests. Evenings were spent planning the next day around dietary limitations and laxatives. My sympathies extended most to the longsuffering person who diligently cleaned my hotel room, who, on the first morning opened the door to discover me "wired" (to a holter tape); the following days brought the increasingly unattractive prospect of insoluble barium and discarded enema sachets.

Friday witnessed an air of relief. Having established bonds of friendship born of sharing common indignities, we left London to await news of the future. It transpired that six people would depart this stage whereas the core of 16 progressed to aeromedical testing at the RAF Institute of Aviation Medicine at Farnborough, Hampshire. This facility houses the only human centrifuge in Britain, a daunting steel and wire machine bearing testament to the quality of engineering of the $1950 \mathrm{~s}$. A pivoted gondola at the end of each of two arms allows subjects to experience high $g$ forces in the $\mathrm{x}$ (vertical) and $\mathrm{z}$ (horizontal) vectors, simulating forces of entry into low earth orbit. My first day began with lower body negative pressure to measure my cardiovascular response to large fluid shifts, before my first exposure to $g z$.

Pooling of blood at high $g$ can lead to various Fymptoms; with falling retinal artery perfusion pressure the peripheral vision becomes veiled by "greyout," until blackout with full consciousness supersedes. This rapidly progresses to hypoxic unconsciousness-pilots' dreaded $g$ lock. Isometric lower limb contraction and vigorous Valsalva manoeuvre $-g$ straining-increases individual tolerance 
considerably, to the extent that 40 seconds of $8 \cdot 2 \mathrm{gz}$ was an exhilarating experience marred only by central anginal chest pain due to sternal pressure: any roller coaster will forever be a tame comparison.

Not so gx however, where the upright posture encourages massive distal pooling and at the final run at 5 gx I rapidly "greyed" then "blacked out," before lapsing into sleep. I awoke feeling extraordinarily tranquil, seemingly hours but in reality only seconds later, with a doctor grinning down at me. The intervening period was blurred by bizarre but not unpleasant memories of complex dreams concerning dogs, mingled with nagging sensations of confusion. The video evidence explained my curious calmness on waking - a hypoxic tonic-clonic fit. I completed my other tests of prolonged altitude simulation and motion sickness satisfactorily, but knew my centrifugal performance was a major setback.

Thus ended my involvement with Juno, in combination with an unacceptable degree of myopia and a history of hay fever. Soviet medical review excluded me and numerous other candidates for various seemingly minor problems. I cannot deny a tinge of envy at those who, having become friends, will continue along the path. I feel a certain self satisfaction, and privilege, to have met a group of fascinating people, one of whom will make history. Although no experience was necessary, much has been gained.

\title{
Hospital formularies: need for continuous intervention
}

\author{
John Feely, Robert Chan, Lelia Cocoman, Kenneth Mulpeter, Patricia O'Connor
}

\begin{abstract}
The effects of introducing a hospital formulary alone and with active intervention were compared prospectively with regard to drug costs and the quality of prescribing. Intervention comprised feedback on prescribing habits, peer comparison, and information on drugs. Aspects of prescribing that were not subjected to intervention did not alter. In the year in which intervention occurred generic prescribing rose by $50 \%$; inappropriate prescribing and overall use of third generation cephalosporins fell; and compliance with the recommended list of drugs was good. Overall, drug costs remained static, compared with a projected increase of $10.25 \mathrm{~m}$; in a comparative control hospital drug costs rose by $18 \%$. During the next year, when no form of intervention took place, previous gains were eroded and drug costs rose.
\end{abstract}

Continuous intervention, review, and feedback are required if a formulary is to continue to achieve its objectives.

\section{Introduction}

Hospital formularies are now widely accepted,' and there is evidence that a restricted drugs policy can initially reduce costs. ${ }^{1-4}$ As there is little information on the effect of a formulary on the quality of prescribing ${ }^{45}$ or the need for continuous intervention we undertook a study in which certain aspects of the formulary and prescribing were selectively subjected to feedback for a defined period and others were not. Drug use was monitored over three years.

\section{Department of}

Pharmacology and

Therapeutics, Trinity

College Medical School, St

James's Hospital, Dublin 8,

Ireland

John Feely, MD, professor

Robert Chan, $\mathrm{MB}$, medical

intern

Lelia Cocoman, BSC,

pharmacist

Kenneth Mulpeter, MRCPI, registrar

Patricia O'Connor, MRCPI, registrar

Correspondence to:

Professor Feely.

\section{Methods}

This hospital is an 800 bed general hospital with expanding services whose drug bill rose by about $20 \%$ a year in the 1980 s to $£ 1.7 \mathrm{~m}$ in 1985 . As there is no Irish national formulary our list of recommended drugs was drawn up after a survey of the drugs actually used, reference to experience with the Dundee formulary, ${ }^{3}$ and information from the coordinating centre for drugs and therapeutics committees in Southampton. ${ }^{6}$ The formulary contained 330 drugs covering all categories, and a preliminary description has been published elsewhere. The aim was to improve prescribing practice by encouraging prescription of drugs on the grounds of efficacy, safety, and economy. Four hundred prescriptions were collected from representative general medical and surgical units on two occasions before the formulary was introduced. Subsequent surveys were made one, four, and 12 months after its introduction; during this year intervention took place.' The final survey was made at the end of a further 12 months during which no intervention occurred. That these samples were representative of overall hospital prescribing was confirmed by two separate surveys of over 4500 prescriptions.

Specific forms of intervention included feedback of prescribing habits to individual prescribers and consultants in charge of units. In addition, peer comparisons and examples of specific savings were provided to prescribers. A drug information note was prepared in conjunction with the hospital's clinical microbiologists on the use and abuse of third generation cephalosporins. In addition, prescribing habits were discussed at the monthly medical committee meetings. Only selected aspects of prescribing were, however, subjected to intervention. Special attention was paid to generic prescribing and the use of third generation cephalosporins. General guidelines on prescribing were contained within the formulary, but otherwise no specific form of intervention was made with regard to using clear instructions, giving dates for the start and end of treatment, using capital letters, and recording drug allergies on prescription charts. When a prescriber wished to obtain a drug not included in the formulary a separate requisition was completed, and these were reviewed on a monthly basis.

We used Dr Steevens's Hospital, with 220 beds, as a concurrent control as it adopted an identical formulary without any form of intervention.

\section{Results}

Generic prescribing-The amount of generic prescribing in the hospital rose progressively by almost $50 \%$ in the year after introduction of the formulary, in both the medical and surgical units (from $50 \%$ to $72 \%$ and $38 \%$ to $62 \%$ respectively). In the subsequent year without feedback generic prescribing reverted to its former level (table I).

Prescribing practice-Aspects of prescribing practice that were not subjected to feedback did not change.

Requisition of drugs not in formulary-Once prescribers became familiar with the formulary requisitions for drugs not in it constituted $5 \%$ of all prescriptions and were mostly for analgesics (dextropropoxyphene), compound antibiotics, cephalosporins, theophylline preparations, diuretics, and 